\title{
Aberrant PTEN expression in response to progesterone reduces endometriotic stromal cell apoptosis
}

\author{
JongYeob Choi ${ }^{1}$, MinWha Jo ${ }^{2}$, EunYoung Lee ${ }^{1}$, Seongsoo Hwang ${ }^{3}$ and DooSeok Choi ${ }^{1}$ \\ 'Infertility Clinic, Department of Obstetrics and Gynecology, Samsung Medical Center, Sungkyunkwan University \\ School of Medicine, Gangnam-gu, Seoul, Korea, ${ }^{2}$ Center for Clinical Research, Samsung Biomedical Research \\ Institute, Gangnam-gu, Seoul, Korea and ${ }^{3}$ Animal Biotechnology Division, National Institute of Animal Science, \\ Wanju-gun, Jeollabuk-do, Korea
}

Correspondence should be addressed to D Choi; Email: dooseok.choi@samsung.com

\begin{abstract}
In some human cancer cells, PTEN (phosphatase and tensin homolog deleted on chromosome 10) is known to regulate autophagy induction positively through the inhibition of PI3K/AKT pathway, leading to the activation of mTOR, a major negative regulator of autophagy. Recent studies reported that PTEN expression is abnormally decreased in endometriotic lesions. In endometriosis, abnormal PTEN expression may contribute to the alteration of endometrial cell autophagy, which may affect apoptosis because endometrial cell autophagy is directly involved in the regulation of apoptosis. To test this hypothesis, we evaluated the involvement of PTEN in the regulation of autophagy induction in human normal endometrial stromal cells (NESCs). In addition, we sought to determine whether aberrant PTEN expression in endometriotic cyst stromal cells (ECSCs) is associated with autophagy dysregulation, and a subsequent decrease in apoptosis. Our results show that PTEN expression was enhanced by progesterone treatment in NESCs. Subsequently, autophagy and apoptosis induction increased through the inhibition of AKT and mTOR activity. This progesteroneinduced increase in apoptosis was reversed by the inhibition of autophagy induction using either mifepristone (progesterone receptor modulator) or PTEN inhibitor. In contrast, progesterone had no significant effects on PTEN expression, AKT, mTOR activity, autophagy or apoptosis in ECSCs. Furthermore, in contrast to normal eutopic endometrium, endometriotic tissues have constant PTEN expression, autophagy and apoptosis throughout the menstrual cycle. In conclusion, our results suggest abnormal PTEN expression in response to progesterone was observed in ECSCs, which led to the dysregulation of autophagy induction via AKT/mTOR signalling and a subsequent decrease in apoptosis.

Reproduction (2017) 153 11-21
\end{abstract}

\section{Introduction}

Endometriosis is characterised by the presence of endometrial tissue outside of the uterine cavity. These lesions typically occur on the ovaries and pelvic peritoneum. Endometriosis is one of the most common causes of chronic pelvic pain, dysmenorrhoea and infertility (Giudice \& Kao 2004, Bulun 2009). It affects approximately $5-15 \%$ of reproductive-age women and $20-50 \%$ of infertile women (Eskenazi \& Warner 1997, Pritts \& Taylor 2003). The pathogenesis of endometriosis is poorly understood. Unfortunately, the current options for management of endometriosis are limited (Kauppila \& Rönnberg 1985, Vercellini et al. 1993, Crosignani et al. 2006). To improve its treatment, it is essential to investigate the molecular mechanisms associated with the development of endometriosis.

Apoptosis is a form of programmed cell death (PCD). Accumulating evidence suggests that reduced apoptosis in refluxed endometrial cells might enhance their survival at ectopic sites, leading to the development of endometriosis (Gebel et al. 1998, Dmowski et al. 2001). However, apoptosis may not be the only mechanism of endometrial cell death. Autophagy, a nonapoptotic form of PCD, is an intracellular bulk degradation system in which a portion of the cytoplasm is enveloped in double membrane-bound structures called autophagosomes. These autophagosomes undergo maturation, and eventually fuse with lysosomes for degradation (Klionsky \& Emr 2000, Levine \& Klionsky 2004). Autophagy also plays an important role in promoting cell death by promoting caspase-dependent apoptosis in normal (Choi et al. 2011a,b) and cancerous cells (Boya et al. 2003, 2005, Wu et al. 2009), although it was originally thought to represent a survival response to nutrient deprivation and other forms of cellular stress (Yorimitsu \& Klionsky 2005). Furthermore, we recently showed that autophagy induction exerts a proapoptotic effect on normal human endometrial cells (Choi et al. 2012). However, autophagy is suppressed in endometriotic cells due to the de-repression of mammalian target 
of rapamycin (mTOR), a major negative regulator of autophagy; this results in decreased endometriotic cell apoptosis (Choi et al. 2014). These findings suggest a direct role of aberrant autophagy induction in the pathogenesis of endometriosis. However, dysregulated autophagy has never been fully evaluated in the context of endometriosis.

Phosphatase and tensin homolog deleted from chromosome 10 (PTEN) is one of the most frequently mutated tumour suppressor genes in human cancers (Steck et al. 1997). PTEN is completely lost or mutated in $>50 \%$ of primary endometrioid endometrial cancers (Sun et al. 2001), and in at least $20 \%$ of precancerous endometrial hyperplasias (Levine et al. 1998, Sun et al. 2001). Furthermore, PTEN loss has also been reported in the malignant transformation of endometriosis (Obata et al. 1998, Sato et al. 2000a,b). Therefore, the loss and mutation of PTEN may contribute to the pathogenesis of endometrial cancer and endometriosis. This suggests that normal PTEN expression is a key factor in the multistep process that determines the cellular fate (survival vs death) of endometrial cells. The main function of PTEN is to inhibit the phosphatidylinositol 3-kinase $(\mathrm{PI} 3 \mathrm{~K}) /$ protein kinase $\mathrm{B}$ (AKT) signalling pathway by removing the phosphate from the D3-phosphate group of phosphoinositide-3, 4, 5-triphosphate (PIP3) (Maehama \& Dixon 1998, Katso et al. 2001). In many normal and cancerous cells, the PI3K/AKT pathway has been shown to negatively regulate autophagy induction by activating mTOR (Corcelle et al. 2009, Chen \& Karantza 2011). This finding suggests that inhibiting AKT activity may promote autophagy by preventing mTOR activation. Therefore, PTEN may play an important role in autophagy regulation by controlling the PI3K/AKT pathway. Indeed, the upregulation of PTEN expression has been observed in some human cancer cells. This upregulation decreases AKT activity by inhibiting the PI3K/AKT pathway, thereby leading to mTOR inactivation and subsequent autophagy induction (Arico et al. 2001, Degtyarev et al. 2008, Fan et al. 2010, Nho \& Hergert 2014). Furthermore, progesterone has been shown to upregulate PTEN expression (GuzelogluKayisli et al. 2003). Increased PTEN expression facilitates the inhibition of AKT activity in human endometrial cells (Mutter et al. 2000a,b). In contrast, some ectopic and eutopic endometrial stromal cells in women with endometriosis exhibit progesterone resistance (Attia et al. 2000, Bulun et al. 2006, Rizner 2009). These findings suggest that PTEN expression may be altered by abnormal responses to progesterone in endometriotic cells. This hypothesis was further supported by previous studies that revealed inappropriate decreases in PTEN expression in endometrial cells from women with endometriosis compared with that in diseasefree women (Govatati et al. 2014, Zhang et al. 2014). However, it is not yet clear whether PTEN is involved in the regulation of autophagy induction in endometrial cells or whether abnormal PTEN expression in response to progesterone in endometriotic cells contributes to autophagy dysregulation and reduced apoptosis.

Here, we evaluated the involvement of PTEN in the regulation of autophagy and apoptosis induction in normal endometrial stromal cells. In addition, we studied whether abnormal PTEN expression in response to progesterone affects autophagy and apoptosis induction in endometriotic stromal cells, which may contribute to the pathogenesis of endometriosis.

\section{Materials and methods}

\section{Tissue collection}

Normal endometrial tissues $(n=20)$ were obtained from premenopausal women undergoing hysterectomies for uterine leiomyomas or pelvic organ prolapse. Ectopic endometriotic tissues $(n=30)$ were obtained from ovarian endometriotic cysts (endometrioma). None of the study participants had taken oral contraceptives or hormonal agents for at least three months before surgery. The average age of the participants was $45.5 \pm 3.4$ years for eutopic endometrial tissues and $32.0 \pm 7.7$ years for ectopic endometrial tissues. The endometrial tissue samples were divided into five categories according to the day of the menstrual cycle: early proliferative (days 1-5), mid-to-late proliferative (days 6-14), early secretory (days 15-18), mid-secretory (days 19-23) and late secretory phase (days 24-28). The menstrual cycle day was established based on the patient's menstrual history. Of the 20 eutopic endometrial samples, four each were early proliferative, late proliferative, early secretory, mid-secretory and late secretory, and 6 each of the 30 ectopic endometrial samples were early proliferative, late proliferative, early secretory, mid-secretory and late secretory phase. All samples were snap-frozen in liquid nitrogen and stored at $-80^{\circ} \mathrm{C}$ for western blot analysis. This study was approved by the Ethical Committee of Samsung Medical Center. Written informed consent was obtained from all participants.

\section{Human normal endometrial and endometriotic cyst stromal cell isolation}

Normal endometrial stromal cells (NESCs) and endometriotic cyst stromal cells (ECSCs) in the proliferative phase were dissociated and purified from eutopic endometrial tissues and ovarian endometriotic tissues respectively, using a published procedure (Ryan et al. 1994) with minor modifications. Tissues were rinsed with PBS. The endometrial lining was dissected from the myometrium, minced and digested with $2 \mathrm{mg} / \mathrm{mL}$ type IV collagenase (Sigma Chemical) at $37^{\circ} \mathrm{C}$ for $60 \mathrm{~min}$ with agitation. Stromal cells were separated from epithelial glands using $70 \mu \mathrm{m}$-pore filters, and then a $45 \mu \mathrm{m}$-pore nylon mesh. Filtered cells were plated in T75 flasks and allowed to adhere for approximately $30 \mathrm{~min}$. The flasks were washed with PBS to remove blood cells and debris. The stromal cells were cultured in Dulbecco's modified Eagle's/F12 medium (DMEM/ F12; Gibco-BRL) supplemented with $10 \%$ foetal bovine serum (FBS, Gibco-BRL), $100 \mathrm{U} / \mathrm{mL}$ penicillin and $100 \mathrm{mg} / \mathrm{mL}$ 
streptomycin (Gibco-BRL) in a humidified atmosphere with $5 \% \mathrm{CO}_{2}$ at $37^{\circ} \mathrm{C}$. The medium was changed every other day. At confluence, the cells were subcultured in 24-well culture plates using $1 \mathrm{~mL}$ of culture medium. Endometrial stromal cell suspension purity was determined by immunostaining with vimentin stromal cell-specific antibodies.

\section{In vitro experiments}

Each experiment with NESCs and ECSCs was performed using cells prepared from eutopic endometrial tissues $(n=4)$ and ovarian endometriotic tissues obtained $(n=4)$ from 8 different patients respectively, for sex steroid, mifepristone or PTEN inhibitor treatment. Subcultured NESCs and ECSCs were seeded at $1 \times 10^{6} \mathrm{cell} / \mathrm{sL}$ in poly-L-lysine-coated nonfluorescent thin-bottom glass culture dishes (MatTek, Ashland, MA, USA). Cells were incubated at $37^{\circ} \mathrm{C}$ in $5 \%$ $\mathrm{CO}_{2}$ in DMEM/F12 supplemented with $10 \%$ FBS, glutamine, HEPES, $100 \mathrm{U} / \mathrm{mL}$ penicillin and $100 \mathrm{mg} / \mathrm{mL}$ streptomycin. At $70-80 \%$ confluence, cultures were treated with serum-free Earle's Balanced Salt Solution (EBSS) medium (Sigma) for serum starvation. To mimic physiological hormonal changes, NESCS and ECSCs were also cultured with EBSS medium before hormone treatment. After $24 \mathrm{~h}$ of culture, either oestradiol (10 nM, Sigma) alone or oestradiol $(10 \mathrm{nM})+$ progesterone ( $1 \mu \mathrm{M}$, Sigma) was added for $72 \mathrm{~h}$. For the pharmacological inhibition experiments, NESCs were cultured in EBSS medium supplemented with oestradiol $(10 \mathrm{nM})$ and progesterone $(1 \mu \mathrm{M})$ for $72 \mathrm{~h}$. Two hours before the analysis, a progesterone receptor modulator $(100 \mathrm{nM}$, mifepristone, Sigma) or PTEN inhibitor (100 nM, SF1670, Sigma) was added to the culture medium to inhibit the effects of progesterone or PTEN respectively. For the apoptosis experiments, NESCs were cultured in EBSS supplemented with oestradiol (10nM) and progesterone $(1 \mu \mathrm{M})$ for $72 \mathrm{~h}$. Next, mifepristone or PTEN inhibitor was added to the culture medium $6 \mathrm{~h}$ before analysis to inhibit the induction of progesterone- or PTENmediated autophagy respectively. Treatments were stopped by removing the medium. Cells were then either harvested by scraping to generate protein extracts or fixed for subsequent immunofluorescence and electron microscopy analyses. Apoptosis of the endometrial stromal cells was evaluated using annexin-V/propidium iodide (PI) staining.

\section{Western blot}

During autophagy induction, LC3 was converted from LC3-I to LC3-II. The LC3-II localises to isolated membranes and autophagosomes (Kabeya et al. 2000, 2004). The level of LC3-II expression has been shown to correlate with the autophagosome number (Nara et al. 2002). Therefore, the level of LC3-II protein was assessed using western blotting as a surrogate measure of endometrial cell autophagy. The level of autophagy is also reflected by the expression level of autophagy-related 5 (ATG5), which is a protein specifically required for autophagy induction. AKT activity was determined by quantifying the phosphorylated (i.e., active) form of AKT. The activity of the mTOR pathway was determined by measuring the phosphorylation of ribosomal protein S6 kinase (S6K). S6K is a direct substrate of mTOR. Therefore, the phosphorylation status of S6K reflects the activity of the mTOR pathway (Sarbassov et al. 2005). The extent of endometrial cell apoptosis was determined by measuring the level of cleaved poly (ADP-ribose) polymerase (PARP) and cleaved caspase-3, the final mediator of apoptosis (Porter \& Janicke 1999). Endometrial tissues, endometriotic tissues or cultured stromal cells were lysed with an ice-cold radioimmunoprecipitation assay buffer that was supplemented with a protease inhibitor cocktail (Sigma). For complete solubilisation of cellular proteins, lysates were incubated on ice for $30 \mathrm{~min}$ and then centrifuged at $13,000 \mathrm{~g}$ at $4{ }^{\circ} \mathrm{C}$ for $30 \mathrm{~min}$. Proteins in whole cell lysates $(20 \mu \mathrm{g} / \mathrm{lane})$ were separated by sodium dodecyl sulphate polyacrylamide gel electrophoresis (SDS-PAGE). The proteins were then transferred to polyvinylidene difluoride membranes (Bio-Rad). After blocking nonspecific binding sites with 5\% skim milk, membranes were incubated with rabbit polyclonal antibodies against PTEN (1:1000; Cell Signaling Technology), total and phosphorylated AKT (Ser473) (1:500 and 1:500 respectively; Cell Signaling Technology), total and phosphorylated S6K (Ser235/236) (1:1000 each; Cell Signaling Technology), ATG5 (1:1000; Cell Signaling Technology), LC3 (1:1000; Cell Signaling Technology) or cleaved caspase-3 (1:1000; Cell Signaling Technology) overnight at $4^{\circ} \mathrm{C}$. Membranes were washed 3 times for $15 \mathrm{~min}$ in wash buffer (PBS containing 0.1\% Tween 20) and incubated with the appropriate secondary antibodies (1:5000; Santa Cruz Biotechnology) at room temperature for $1 \mathrm{~h}$. After three 15-min washes in wash buffer at room temperature, immunoreactive bands were detected using an enhanced chemiluminescence kit (Amersham Pharmacia Biotech). Bands were quantified using NIH Image) software (NIH Image Processing and Analysis in Java). The expression levels of LC3-II and cleaved caspase-3 were normalised to that of $\beta$-actin. In contrast, the expression levels of phosphorylated AKT and S6K were normalized to those of total AKT and S6K respectively.

\section{Immunofluorescence}

Endometrial stromal cells were cultured on sterilised glass coverslips, fixed with $4 \%$ paraformaldehyde and blocked with $0.1 \%$ bovine serum albumin in PBS. Cells were incubated with anti-LC3 mouse polyclonal antibody (1:500) and PTEN rabbit polyclonal antibody (1:500) in PBS. Next, the cells were incubated with Alexa 488- and 568-conjugated secondary antibodies (1:5000; Vector Laboratories, Burlingame, CA, USA). The slides were mounted in mounting media (Vector Laboratories) with 4',6-diamidino-2-phenylindole (DAPI) for nuclear staining. Images were captured with a confocal microscope (Bio-Rad).

\section{Transmission electron microscopy}

To identify autophagosomes at the ultrastructural level, endometrial stromal cells were fixed with $2.5 \%$ glutaraldehyde in $0.1 \mathrm{M}$ cacodylate buffer $(\mathrm{pH} 7.4)$ for $45 \mathrm{~min}$ at $4^{\circ} \mathrm{C}$. The cells were then rinsed in cacodylate buffer, post-fixed in $1 \% \mathrm{OsO}_{4}$ in cacodylate buffer, dehydrated and embedded in Eponate. Ultra-thin sections were briefly contrasted with 
uranyl acetate and photographed with a transmission electron microscope (Hitachi 7100).

\section{Assessment of human endometrial stromal cell apoptosis}

Apoptotic cell percentages were determined with an annexinV-fluorescein isothiocyanate (FITC) apoptosis detection kit (BD Biosciences, San Diego, CA, USA) according to the manufacturer's protocol. Briefly, after drug treatment, $1 \times 10^{5}$ cells were pelleted and washed once with PBS. Cells were then resuspended in $100 \mu \mathrm{L}$ binding buffer $(10 \mathrm{mM}$ HEPES $\mathrm{pH} 7.4$, $150 \mathrm{mM} \mathrm{NaCl}, 5 \mathrm{mM}$ potassium chloride, $1 \mathrm{mM} \mathrm{MgCl} 2$, and $2 \mathrm{mM}$ calcium chloride). Subsequently, $5 \mu \mathrm{L}$ annexin- $\mathrm{V}$ and $\mathrm{PI}$ were added and the cells were incubated for $15 \mathrm{~min}$ at room temperature in the darkness. After incubation, $400 \mu \mathrm{L}$ binding buffer was added, and cells were analysed using a FACSAria flow cytometry (BD Biosciences, Heidelberg, Germany). At least 10,000 cells were analysed before treatment. Data analysis was conducted using CellQuest software.

\section{Statistical analysis}

Relative protein expression levels and proportions of apoptotic cells are reported as means \pm standard errors. All statistical analyses were performed using ANOVA. Significant differences between the treatment groups were identified using Duncan's multiple range test. $P$ values $<0.05$ were considered statistically significant.

\section{Results}

Upregulated PTEN expression increases endometrial cell autophagy by inhibiting AKT activity, thereby inactivating $\mathrm{mTOR}$

We sought to determine the involvement of PTEN in the regulation of autophagy induction in endometrial cells through its control of AKT and mTOR activity. To do so, we characterised the effects of oestrogen and progesterone on the levels of PTEN, phosphorylated AKT, S6K, ATG5 and LC3-II expression in cultured NESCs. Progesterone treatment significantly increased PTEN, LC3-II and ATG5 expression in oestrogen-treated NESCs $(P<0.05)$ (Fig. $1 \mathrm{~A}$ and B). In contrast, AKT and S6K phosphorylation were both significantly decreased after progesterone treatment $(P<0.05)$. To block the effects of progesterone, we used pharmacological inhibitors of progesterone and PTEN and measured the levels of phosphorylated AKT, S6K and LC3-II expression. Progesterone-stimulated PTEN expression was suppressed by the addition of both progesterone receptor modulator (mifepristone) and PTEN inhibitor (SF1670). This suppression was accompanied by increased AKT and S6K phosphorylation, and decreased LC3-II and ATG5 expression $(P<0.05)$ (Fig. $1 \mathrm{~A}$ and B).

Next, we used immunofluorescence staining to determine the subcellular localisations of endogenous
PTEN and LC3. The distributions of endogenous PTEN and LC3 were readily detected in the cultured NESCs as green and red fluorescent areas respectively (Fig. 1C). In NESCs cultured with oestrogen alone, there was weak PTEN staining and a few punctate LC3-II structures were detected (Fig. 1C, Est). The addition of progesterone resulted in intense PTEN immunoreactivity and the cytoplasmic accumulation of punctate LC3-II structures (Fig. 1C, Est + Prog). This change could be prevented by the addition of either mifepristone (Fig. 1C, Est + Prog + Mife) or a PTEN inhibitor (Fig. 1C, Est+Prog+PTEN In). To confirm these observations on an ultrastructural level, we analysed the autophagosome formation using transmission electron microscopy. Autophagic structures are characterised by multiple autophagosomes, which are double-membraned vacuoles containing engulfed cytoplasmic material. In the NESCs cultured with oestrogen, there was an accumulation of autophagic vacuoles (indicative of autophagy induction) after the addition of progesterone. However, this accumulation could also be suppressed by the addition of either mifepristone or a PTEN inhibitor (Fig. 1D).

\section{Endometrial cell apoptosis is induced after increased PTEN expression}

To determine whether PTEN-mediated autophagy induction in NESCs drives apoptosis, we evaluated the effects of progesterone, mifepristone and SF1670 on apoptosis in oestrogen-treated NESCs. The NESCs treated with progesterone exhibited significantly higher expression of cleaved PARP and cleaved caspase- 3 than did the NESCs cultured in oestrogen alone (Fig. 2A and B). Progesterone-mediated upregulation of cleaved PARP and cleaved caspase-3 expression was decreased by the addition of mifepristone and by the addition of SF1670. We also conducted flow cytometry assays using annexin- $\mathrm{V}$ and $\mathrm{PI}$ to determine the proportion of apoptotic cells. The proportion of apoptotic NESCs induced by treatment with oestrogen alone increased significantly (by 2.23-fold) in the presence of progesterone (Fig. 2C and D). In contrast, the proportion of apoptotic progesterone-treated NESCs decreased by $\sim 42 \%$ and $\sim 30 \%$ after the addition of mifepristone and SF1670 respectively $(P<0.05)$.

\section{The effects of oestrogen and progesterone on PTEN expression, AKT levels, mTOR activity, autophagy and apoptosis in NESCs and ECSCs in vitro}

We sought to determine whether the effects of ovarian steroids on PTEN expression, AKT and mTOR activity are different in NESCs and ECSCs. In addition, we studied whether this differential expression affects autophagy and apoptosis in ECSCs. To do so, we characterised the effects of oestrogen and progesterone on the levels of PTEN, phosphorylated AKT, S6K, ATG5, LC3-II and 

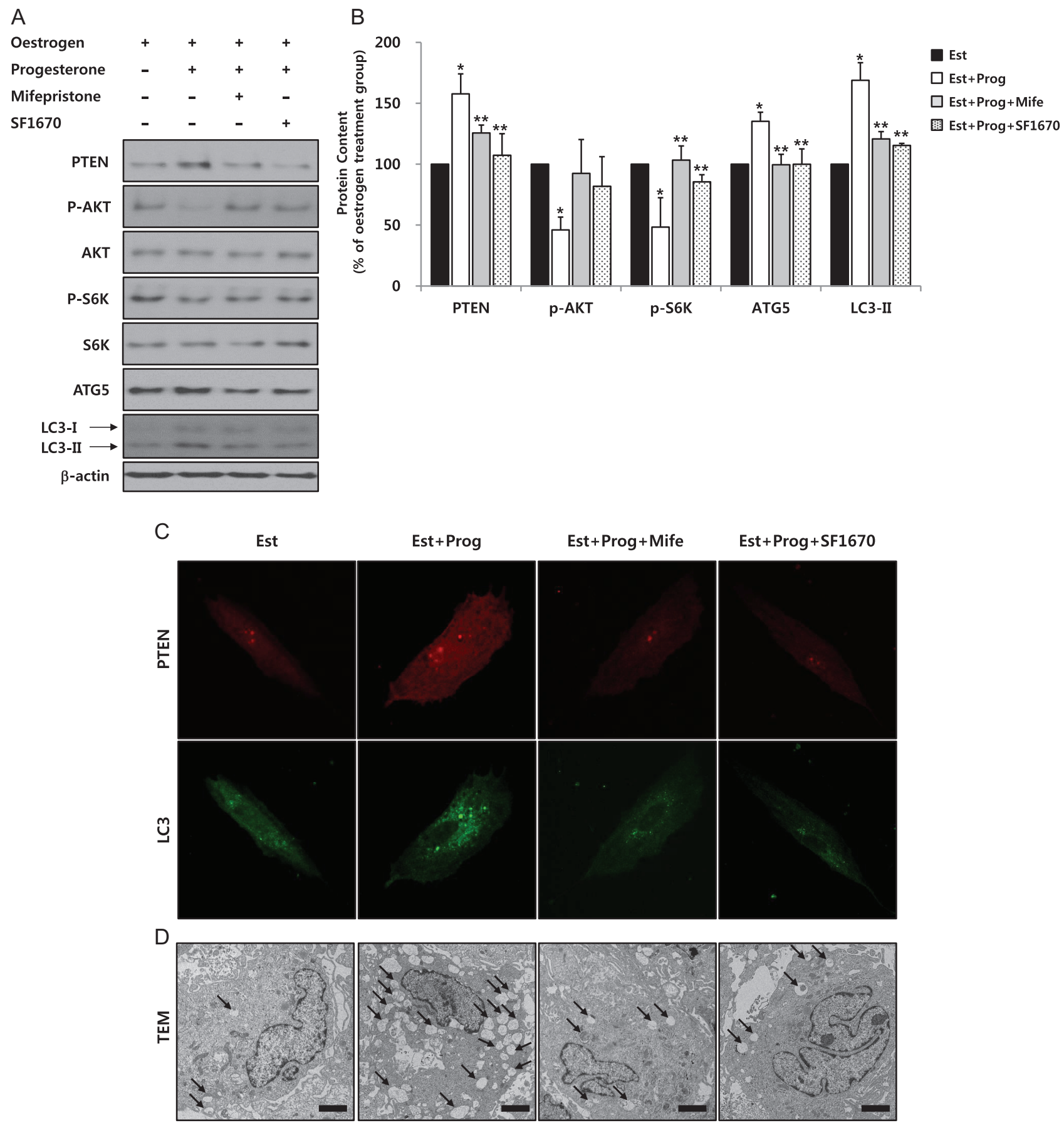

Figure 1 Effects of oestrogen and progesterone on the levels of PTEN, phosphorylated AKT, phosphorylated S6K, ATG5 and LC3-II in NESCs. (A) Representative immunoblots of PTEN, AKT, S6K, ATG5 and LC3 in protein extracts from in vitro-cultured NESCs. (B) Densitometric quantification of PTEN, phosphorylated AKT (p-AKT), p-S6K, ATG5 and LC3-II protein contents. Experiments were repeated four times and data are expressed as means \pm S.E.S. ${ }^{*} P<0.05$ compared with the oestrogen-only group. ${ }^{*} P<0.05$ compared with the oestrogen + progesterone group. Est, oestrogen; Prog, progesterone; Mife, mifepristone. (C) Double-immunofluorescence staining for PTEN and LC3 in cultured HECs. PTEN and LC3 were stained with red and green fluorophores, respectively. LC3-I exhibited a diffuse cytoplasmic distribution. LC3-II was found in punctate structures. (D) Transmission electron microscope images of NESCs cultured with oestrogen alone, oestrogen + progesterone, oestrogen + progesterone + mifepristone or oestrogen + progesterone +SF1670. Arrows indicate representative autophagosomes. Scale bars: $2 \mu \mathrm{m}$. $\mathrm{N}$, nucleus.

cleaved caspase-3 expression in cultured NESCs and ECSCs. There was significantly higher PTEN expression and decreased phosphorylation of AKT and S6K in NESCs cultured with oestrogen and progesterone compared with that of those cultured with oestrogen alone (Fig. 3A and $B, P<0.05)$. After incubation with oestrogen and 

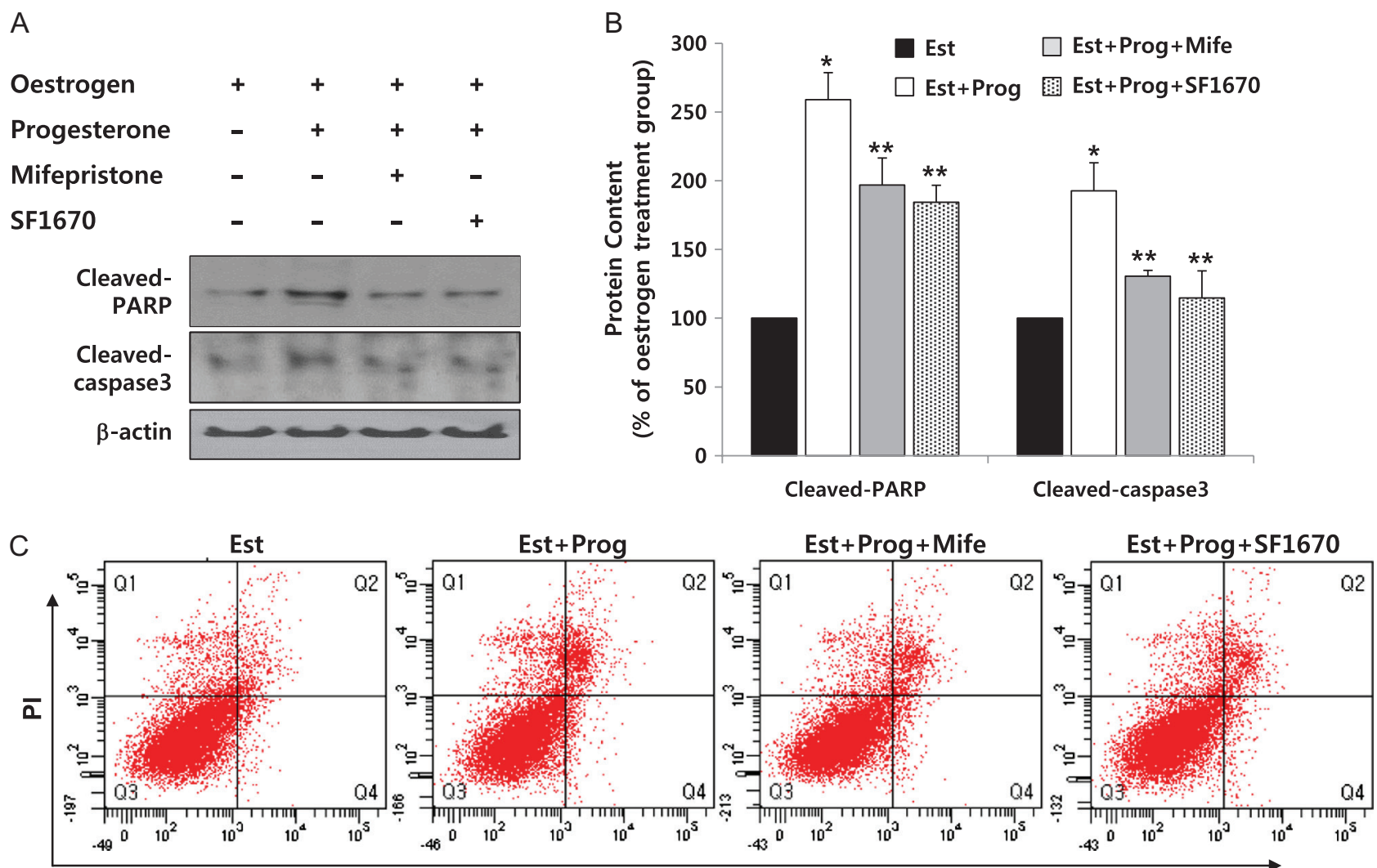

Annexin V

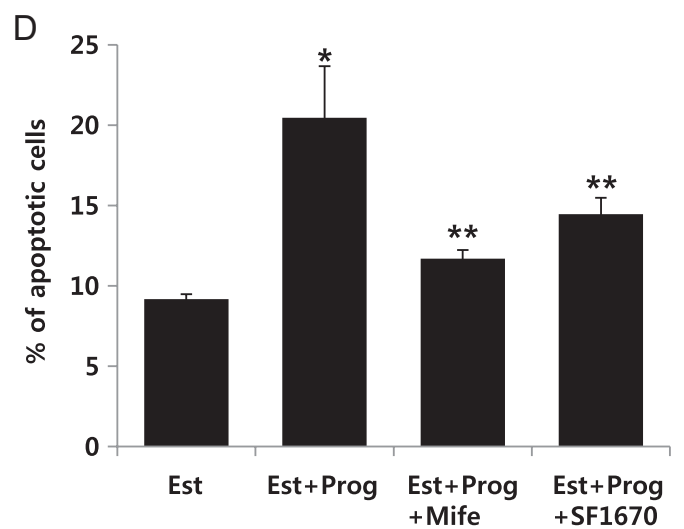

Figure 2 PTEN-mediated autophagy induction increases apoptotic cell death in NESCs. (A and B) Representative immunoblots of cleaved PARP and cleaved caspase 3 in protein extracts from in vitro cultured NESCs. Experiments were repeated four times and data are expressed as means \pm standard errors (S.E.S). ${ }^{*} P<0.05$ compared with the oestrogen-only group. ${ }^{* *} P<0.05$ compared with the oestrogen + progesterone group. Est, oestrogen; Prog, progesterone; Mife, mifepristone. (C) Representative flow cytometry plots from the oestrogen alone, oestrogen + progesterone, oestrogen + progesterone + mifepristone and oestrogen + progesterone +SF1670 groups. Lower right quadrant, annexin $\mathrm{V}+/ \mathrm{PI}-$; upper right quadrant, annexin $\mathrm{V}+/ \mathrm{PI}+$ (considered apoptotic). (D) Percentages of apoptotic cells from flow cytometry. Bar graphs display means \pm standard errors (S.E.S). ${ }^{*} P<0.05$ compared with the oestrogen-only group. ${ }^{* *} P<0.05$ compared with the oestrogen + progesterone group.

progesterone, the removal of both hormones from the media for $24 \mathrm{~h}$ also increased PTEN expression and decreased phosphorylated AKT and S6K expression $(P<0.05)$. Furthermore, the expression levels of LC3-II and cleaved caspase- 3 increased significantly in NESCs cultured with the addition of progesterone or withdrawal of both oestrogen and progesterone compared with these respective levels in NESCs cultured with oestrogen alone $(P<0.05)$. In ECSCs, however, the oestrogen and progesterone treatment did not influence the expression of PTEN, phosphorylated AKT, S6, LC3-II or cleaved caspase-3 (Fig. 3C). 

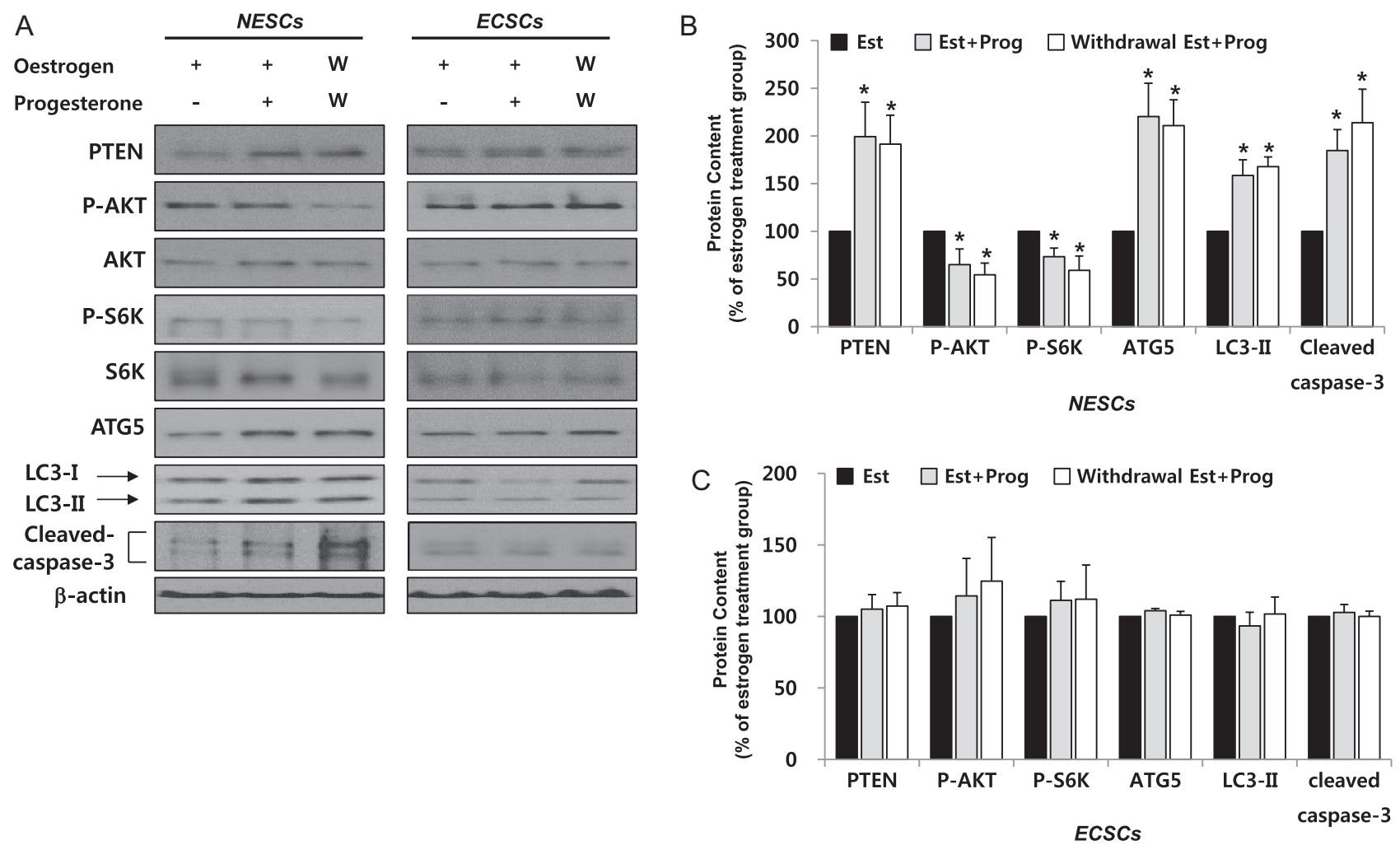

Figure 3 Differential expression of PTEN, autophagy and apoptosis in NESCs and ECSCs following treatment with oestrogen and/or progesterone. (A) Representative immunoblots of PTEN, phosphorylated AKT and S6K, ATG5, LC3 and cleaved caspase-3 in NESCs (left) and ECSCs (right). (B and C) Densitometric quantification of PTEN, phosphorylated AKT and S6K, ATG5, LC3-II and cleaved caspase-3 protein in NESCs (B) and ECSCs (C). Experiments were repeated four times, and data are expressed as mean $( \pm$ S.E.). * Significant differences $(P<0.05)$ when compared with the oestrogen alone group. W, withdrawal.

\section{PTEN expression, AKT levels, mTOR activity, autophagy and apoptosis induction in normal eutopic endometrium and ectopic endometriotic tissues during the menstrual cycle}

We investigated whether PTEN, AKT and mTOR activity are expressed differently during the menstrual cycle in normal eutopic endometrium and ectopic endometriotic tissues from ovarian endometriotic cysts. There PTEN expression was higher during the late proliferative phase than it was during the early proliferative phase, although the difference was not significant (Fig. 4A and B). The PTEN expression was significantly higher in the early (1.54-fold), mid- (1.65-fold) and late-(1.79-fold) secretory endometrium than it was in the early proliferative endometrium. In contrast, the levels of phosphorylated AKT and S6K were significantly lower in the secretory phase than they were in the early proliferative phase $(P<0.05)$. We also investigated whether PTEN-mediated endometrial AKT and mTOR activity is associated with autophagy and apoptosis induction during the menstrual cycle. To do so, we evaluated the expression patterns of Atg5, LC3-II and cleaved caspase-3. In the endometrial cells, the expression levels of Atg5, LC3-II and cleaved caspase-3 increase significantly during the secretory phase, peaking during the late secretory phase $(P<0.05$; Fig. 4A and B).
In ectopic endometriotic tissues, however, the expression levels of PTEN, phosphorylated AKT, S6K, LC3-II and cleaved caspase-3 did not change during the secretory phase, instead remaining constant throughout the menstrual cycle (Fig. 4C). We also compared the expression levels of PTEN, phosphorylated AKT, S6K, LC3-II and cleaved caspase-3 during the late secretory phase of the menstrual cycle. The expression levels of PTEN, LC3-II and cleaved caspase-3 were significantly lower in the ectopic endometriotic tissues than they were in the normal endometrium; in contrast, the levels of phosphorylated AKT and S6K were significantly higher $(P<0.05)$ (Fig. 4D and E).

\section{Discussion}

In some human cell lines, the tumour suppressor PTEN positively regulates autophagy by inhibiting the PI3K/ AKT/mTOR pathway (Arico et al. 2001, Degtyarev et al. 2008). Although PTEN has been reported to inhibit the $\mathrm{PI} 3 \mathrm{~K} / \mathrm{AKT}$ pathway in human endometrial cells (Mutter et al. 2000a,b), its precise role in endometrial cell autophagy has remained unclear. Here, we evaluated whether PTEN is involved in autophagy induction by controlling the PI3K/AKT/mTOR pathway in human endometrial cells. In human endometrial cells, PTEN 

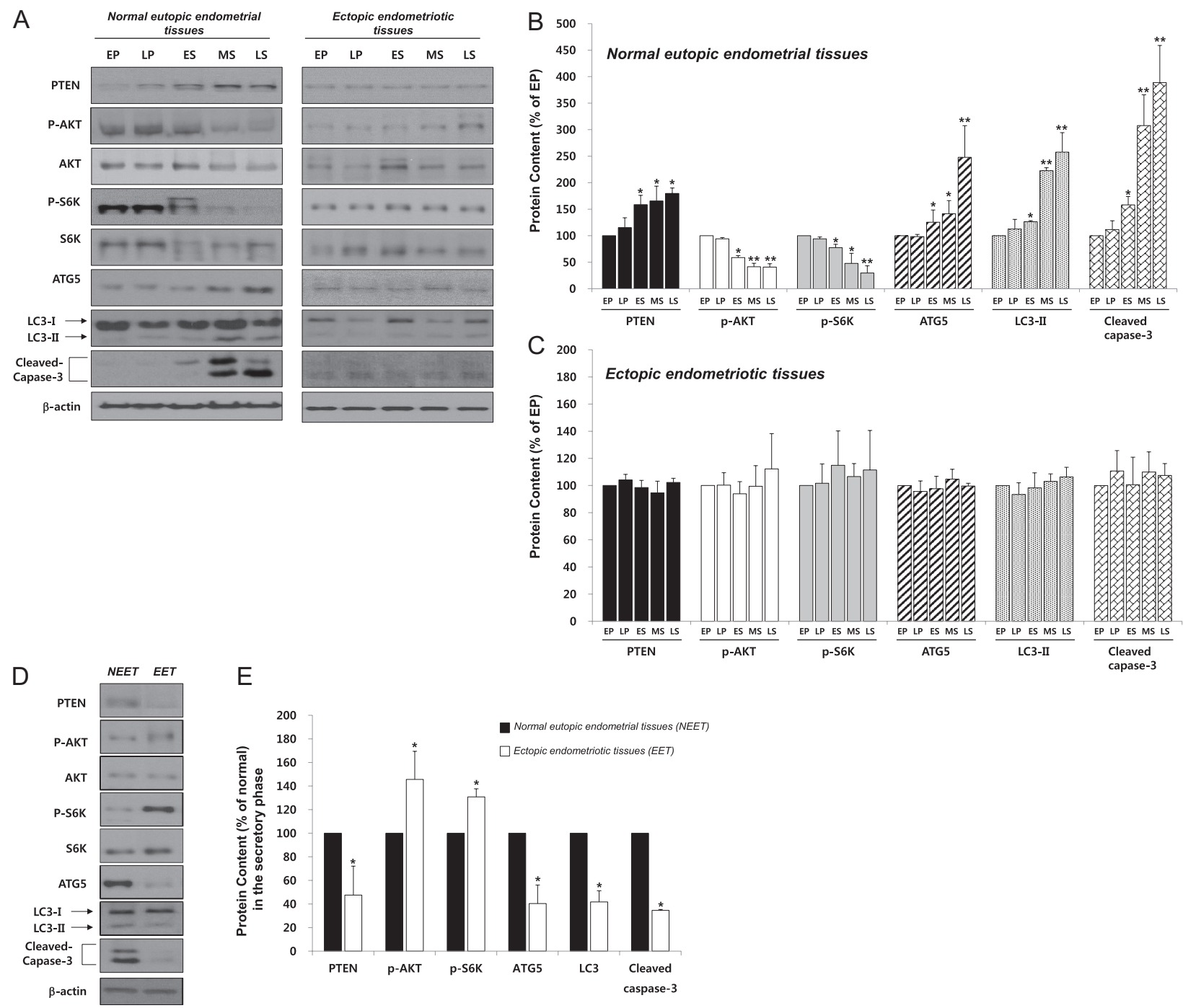

Figure 4 Expression of PTEN, phosphorylated AKT and S6K, ATG5, LC3-II and cleaved caspase-3 in normal eutopic endometrial and ectopic endometriotic tissues during the menstrual cycle. (A) Representative immunoblots of PTEN, phosphorylated AKT and S6K, ATG5, LC3 and cleaved caspase-3 in eutopic endometrial (left) and ectopic endometriotic tissues (right). (B and C) Densitometric quantification of PTEN, phosphorylated AKT and S6K, ATG5, LC3 and cleaved caspase-3 protein contents in eutopic endometrial (B) and ectopic endometriotic tissues (C). EP, early proliferative phase; ES, early secretory phase; LP, mid- to late-proliferative phase; LS, late-secretory phase; MS, mid-secretory phase. Data are mean \pm S.E. ${ }^{*} P<0.05$ compared with EP. ${ }^{* *} P<0.05$ compared with ES. (D and E) Comparison of PTEN, phosphorylated AKT and S6K, ATG5, LC3 and cleaved caspase-3 in late secretory normal eutopic endometrial (NEET) and ectopic endometriotic tissues (EET).

Representative immunoblots (D) and densitometric quantification (E) of PTEN, phosphorylated AKT and S6K, ATG5, LC3 and cleaved caspase-3. Experiments were repeated four times, and data are expressed as means \pm S.E.S. ${ }^{*} P<0.05$ compared with normal.

expression (Guzeloglu-Kayisli et al. 2003) and autophagy induction (Choi et al. 2014) have been described to display a progesterone-dependent expression pattern. Similarly, our in vitro experiments showed that oestrogen treatment of NESCs results in increased expression of PTEN and LC3-II. After the addition of progesterone, there is downregulation of phosphorylated AKT and S6K. These findings suggest that progesterone promotes autophagy induction in endometrial cells by increasing PTEN expression, which then inhibits AKT and mTOR activity. Our pharmacological data support this finding. Specifically, treatment with mifepristone (a potent progesterone receptor modulator) or a PTEN inhibitor downregulates the expression of PTEN and LC3-II and promotes AKT and mTOR activity. Both mifepristone and PTEN inhibitors block the effects of progesterone treatment. These results indicate that progesterone-mediated PTEN expression increases autophagy induction in NESCs via the AKT/ mTOR signalling. Immunofluorescence analysis also supported the hypothesis that progesterone enhances autophagy induction by regulating PTEN expression in NESCs. Specifically, the accumulation of LC3-II was accompanied by increased PTEN expression. 
Furthermore, our TEM images provided direct evidence that autophagy induction in progesterone-treated NESCs decreased by the addition of mifepristone and by the addition of a PTEN inhibitor. Specifically, ultrastructural changes typical of autophagy, such as decreased numbers of autophagosomes within cells, were evident. These results suggest that progesterone increases PTEN expression, which then is directly involved in autophagy induction through the regulation of AKT and mTOR activity in human endometrial stromal cells.

According to previous studies, autophagy is an important cellular mechanism responsible for apoptosis induction in some systems (Saiki et al. 2011, Chow et al. 2012, Singh et al. 2012). Consistent with this finding, we have shown that autophagy induction is closely related to the induction of apoptosis in both endometrial and endometriotic cells (Choi et al. 2014). Indeed, autophagy induction promoted apoptosis; its proapoptotic effect was reversed by autophagy inhibition with 3-methyladenine (3-MA, autophagy inhibitor). Therefore, the high expression levels of PTEN in the progesterone-treated endometrial cells in this study may promote apoptotic cell death by enhancing autophagy induction. This hypothesis is supported by the observation that the progesterone-induced increase in apoptosis was prevented by mifepristone or a PTEN inhibitor. These results indicate that progesterone-induced PTEN expression plays a pivotal role in the regulation of apoptosis by controlling autophagy induction in endometrial cells. In contrast, it is well known that endometriosis is associated with progesterone resistance (Attia et al. 2000, Bulun et al. 2006, Rizner 2009). Indeed, recent reports have suggested that endometriotic cells have an abnormal response to ovarian steroids, which suppresses autophagy and apoptosis induction during the secretory phase of the menstrual cycle (Choi et al. 2014). Therefore, it is possible that abnormal PTEN response to progesterone in endometriotic cells may cause autophagy dysregulation, and subsequently decreased apoptosis during the menstrual cycle.

To confirm this hypothesis, we cultured NESCs and ECSCs in vitro with or without the ovarian steroids oestrogen and progesterone to mimic physiologic hormonal changes. We previously reported that these ovarian steroids control autophagy in endometrial cells during the menstrual cycle. These steroids are the two central balancing factors in the human endometrium (Choi et al. 2014). Indeed, autophagy induction in endometrial cells treated with oestrogen alone (the proliferative phase) increased after the addition of progesterone (early-to-mid secretory phase) or with the removal of oestrogen and progesterone (late secretory and menstrual phase). This study further demonstrated that PTEN expression increased with the downregulation of AKT and mTOR activity in oestrogen-treated NESCs by the addition of progesterone and the removal of oestrogen and progesterone. In contrast, progesterone treatment did not affect the PTEN expression in oestrogen-treated ECSCs. This suggests that aberrant PTEN expression in endometriotic cells may be associated with an abnormal response to progesterone, called progesterone resistance. Furthermore, constant PTEN expression was accompanied by constant levels of AKT, mTOR activity, autophagy and apoptosis in ECSCs after ovarian steroid treatment. Accordingly, endometriotic cell autophagy combined with apoptosis may not be induced due to an abnormal PTEN response to progesterone during the menstrual cycle.

These findings were further demonstrated by our in vivo experiment, which evaluated whether PTEN expression, AKT and mTOR activity are expressed differently in human normal endometrium and endometriotic tissues according to the phases of the menstrual cycle. In human endometrial cells, PTEN displays a cycle-dependent expression pattern (Guzeloglu-Kayisli et al. 2003). Specifically, PTEN expression increases as the menstrual cycle progresses, reaching a maximum during the late secretory phase. In this study, we confirmed that PTEN expression increases as the menstrual cycle progresses in endometrial tissues. We also showed that this pattern is inversely correlated with AKT and S6K phosphorylation. Furthermore, we showed that the cyclic change of LC3-II and cleaved capase-3 expression coincides with that of PTEN. These results indicate that the increased PTEN expression enhances endometrial cell autophagy. This occurs through the inhibition of AKT/mTOR signalling, followed by an increase in apoptosis during the secretory phase of the human endometrial cycle. In endometriotic tissues, however, our results also demonstrated that no cyclic changes were observed in the expression patterns of PTEN, phosphorylated AKT, S6K, LC3-II and cleaved caspase-3 throughout the menstrual cycle. Therefore, the constant level of PTEN expression in endometriotic tissues contributes to the dis-inhibition of AKT and mTOR activity, which is related to decreased autophagy and apoptosis. Indeed, PTEN expression, autophagy and apoptosis induction are significantly lower in endometriotic tissues and the activity of AKT and mTOR are higher when comparing the normal endometrium with endometriotic tissues during the late secretory phase. These in vivo results also suggest that endometriotic cell autophagy is suppressed by abnormal PTEN expression during the menstrual cycle, followed by a decrease in apoptosis. Therefore, it can be postulated that altered induction of endometriotic cell autophagy by aberrant PTEN expression facilitates the decreased apoptosis found in endometriotic tissues compared with that in normal tissues.

In conclusion, PTEN is directly involved in autophagy induction via control of AKT/mTOR signalling in normal endometrial cells during the menstrual cycle. However, endometriotic stromal cells exhibit an abnormal response to progesterone, which leads to suppression of PTEN. This suppression results in autophagy inhibition 
via AKT/mTOR signalling, ultimately leading to decreased apoptosis. This pathway may contribute to the pathogenesis of endometriosis.

\section{Declaration of interest}

The authors declare that there is no conflict of interest that could be perceived as prejudicing the impartiality of the research reported.

\section{Funding}

This study was supported by a grant from Samsung Biomedical Research Institute.

\section{References}

Arico S, Petiot A, Bauvy C, Dubbelhuis PF, Meijer AJ, Codogno P \& Ogier-Denis E 2001 The tumor suppressor PTEN positively regulates macroautophagy by inhibiting the phosphatidylinositol 3-kinase/protein kinase B pathway. Journal of Biological Chemistry 276 35243-35246. (doi:10.1074/jbc.C100319200)

Attia GR, Zeitoun K, Edwards D, Johns A, Carr BR \& Bulun SE 2000 Progesterone receptor isoform $A$ but not $B$ is expressed in endometriosis. Journal of Clinical Endocrinology and Metabolism 85 2897-2902. (doi:10.1210/jc.85.8.2897)

Boya P, Gonzalez-Polo RA, Poncet D, Andreau K, Vieira HL, Roumier T, Perfettini JL \& Kroemer G 2003 Mitochondrial membrane permeabilization is a critical step of lysosomeinitiated apoptosis induced by hydroxychloroquine. Oncogene 22 3927-3936. (doi:10.1038/ sj.onc.1206622)

Boya P, González-Polo RA, Casares N, Perfettini JL, Dessen P, Larochette N, Métivier D, Meley D, Souquere S, Yoshimori T et al. 2005 Inhibition of macroautophagy triggers apoptosis. Molecular and Cellular Biology 25 1025-1040. (doi:10.1128/MCB.25.3.1025-1040.2005)

Bulun SE, Cheng YH, Yin P, Imir G, Utsunomiya H, Attar E, Innes J \& Julie Kim J 2006 Progesterone resistance in endometriosis: link to failure to metabolize estradiol. Molecular and Cellular Endocrinology 248 94-103. (doi:10.1016/j.mce.2005.11.041)

Bulun SE 2009 Endometriosis. New England Journal of Medicine 360 268-279. (doi:10.1056/NEJMra0804690)

Chen N \& Karantza V 2011 Autophagy as a therapeutic target in cancer. Cancer Biology and Therapy 11 157-168. (doi:10.4161/cbt.11.2.14622)

Choi J, Jo M, Lee E \& Choi D 2011a Induction of apoptotic cell death via accumulation of autophagosomes in rat granulosa cells. Fertility and Sterility 95 1482-1486. (doi:10.1016/j.fertnstert.2010.06.006)

Choi J, Jo M, Lee E \& Choi D 2011 b The role of autophagy in corpus luteum regression in the rat. Biology of Reproduction 85 465-472. (doi:10.1095/ biolreprod.111.091314)

Choi J, Jo M, Lee E, Oh YK \& Choi D 2012 The role of autophagy in human endometrium. Biology of Reproduction 86 1-10. (doi:10.1095/ biolreprod.111.096206)

Choi J, Jo M, Lee E, Kim HJ \& Choi D 2014 Differential induction of autophagy by mTOR is associated with abnormal apoptosis in ovarian endometriotic cysts. Molecular Human Reproduction 20 309-317. (doi:10.1093/molehr/gat091)

Chow SE, Chen YW, Liang CA, Huang YK \& Wang JS 2012 Wogonin induces cross-regulation between autophagy and apoptosis via a variety of Akt pathway in human nasopharyngeal carcinoma cells. Journal of Cellular Biochemistry 113 3476-3485. (doi:10.1002/jcb.24224)

Corcelle EA, Puustinen P \& Jaattela M 2009 Apoptosis and autophagy: targeting autophagy signalling in cancer cells - 'trick or treats'? FEBS Journal 276 6084-6096. (doi:10.1111/j.1742-4658.2009.07332.x)

Crosignani P, Olive D, Bergqvist A \& Luciano A 2006 Advances in the management of endometriosis: an update for clinicians. Human Reproduction Update 12 179-189. (doi:10.1093/humupd/dmi049)
Degtyarev M, De Mazière A, Orr C, Lin J, Lee BB, Tien JY, Prior WW, van Dijk S, Wu H, Gray DC et al. 2008 Akt inhibition promotes autophagy and sensitizes PTEN-null tumors to lysosomotropic agents. Journal of Cell Biology 183 101-116. (doi:10.1083/jcb.200801099)

Dmowski WP, Ding J, Shen J, Rana N, Fernandez BB \& Braun DP 2001 Apoptosis in endometrial glandular and stromal cells in women with and without endometriosis. Human Reproduction 16 1802-1808. (doi:10.1093/humrep/16.9.1802)

Eskenazi B \& Warner ML 1997 Epidemiology of endometriosis. Obstetrics and Gynecology Clinics of North America 24 235-258. (doi:10.1016/ S0889-8545(05)70302-8)

Fan QW, Cheng C, Hackett C, Feldman M, Houseman BT, Nicolaides T, Haas-Kogan D, James CD, Oakes SA, Debnath J et al. 2010 Akt and autophagy cooperate to promote survival of drug-resistant glioma. Science Signaling 3 ra81. (doi:10.1126/scisignal.2001017)

Gebel HM, Braun DP, Tambur A, Frame D, Rana N \& Dmowski WP 1998 Spontaneous apoptosis of endometrial tissue is impaired in women with endometriosis. Fertility and Sterility 69 1042-1047. (doi:10.1016/S00150282(98)00073-9)

Giudice LC \& Kao LC 2004 Endometriosis. Lancet 364 1789-1799. (doi:10.1016/S0140-6736(04)17403-5)

Govatati S, Kodati VL, Deenadayal M, Chakravarty B, Shivaji S \& Bhanoori M 2014 Mutations in the PTEN tumor gene and risk of endometriosis: a case-control study. Human Reproduction 29 324-336. (doi:10.1093/humrep/det387)

Guzeloglu-Kayisli O, Kayisli UA, AI-Rejjal R, Zheng W, Luleci G \& Arici A 2003 Regulation of PTEN (phosphatase and tensin homolog deleted on chromosome 10) expression by estradiol and progesterone in human endometrium. Journal of Clinical Endocrinology and Metabolism $\mathbf{8 8 0}$ 5017-5026. (doi:10.1210/jc.2003-030414)

Kabeya Y, Mizushima N, Ueno T, Yamamoto A, Kirisako T, Noda T, Kominami E, Ohsumi Y \& Yoshimori T 2000 LC3, a mammalian homologue of yeast Apg8p, is localized in autophagosome membranes after processing. EMBO Journal 19 5720-5728. (doi:10.1093/emboj/19.21.5720)

Kabeya Y, Mizushima N, Yamamoto A, Oshitani-Okamoto S, Ohsumi Y \& Yoshimori T 2004 LC3, GABARAP and GATE16 localize to autophagosomal membrane depending on form-II formation. Journal of Cell Science 117 2805-2812. (doi:10.1242/jcs.01131)

Katso R, Okkenhaug K, Ahmadi K, White S, Timms J \& Waterfield MD 2001 Cellular function of phosphoinositide 3-kinases: implications for development, homeostasis, and cancer. Annual Review of Cell and Developmental Biology 17 615-675. (doi:10.1146/annurev. cellbio.17.1.615)

Kauppila A \& Rönnberg L 1985 Naproxen sodium in dysmenorrhea secondary to endometriosis. Obstetrics and Gynecology 65 379-383.

Klionsky DJ \& Emr SD 2000 Autophagy as a regulated pathway of cellular degradation. Science 290 1717-1721. (doi:10.1126/ science.290.5497.1717)

Levine B \& Klionsky DJ 2004 Development by self-digestion: molecular mechanisms and biological functions of autophagy. Developmental Cell 6 463-477. (doi:10.1016/S1534-5807(04)00099-1)

Levine RL, Cargile CB, Blazes MS, van Rees B, Kurman RJ \& Ellenson LH. 1998 PTEN mutations and microsatellite instability in complex atypical hyperplasia, a precursor lesion to uterine endometrioid carcinoma. Cancer Research 58 3254-3258.

Maehama T \& Dixon JE 1998 The tumor suppressor, PTEN/MMAC1, dephosphorylates the lipid second messenger, phosphatidylinositol 3,4,5-trisphosphate. Journal of Biological Chemistry 273 13375-13378. (doi:10.1074/jbc.273.22.13375)

Mutter GL, Lin MC, Fitzgerald JT, Kum JB \& Eng C 2000a Changes in endometrial PTEN expression throughout the human menstrual cycle. Journal of Clinical Endocrinology and Metabolism 85 2334-2338. (doi:10.1210/jcem.85.6.6652)

Mutter GL, Lin MC, Fitzgerald JT, Kum JB, Baak JP, Lees JA, Weng LP \& Eng C 2000b Altered PTEN expression as a diagnostic marker for the earliest endometrial precancers. Journal of the National Cancer Institute 92 924-930. (doi:10.1093/jnci/92.11.924)

Nara A, Mizushima N, Yamamoto A, Kabeya Y, Ohsumi Y \& Yoshimori T 2002 SKD1 AAA ATPase-dependent endosomal transport is involved in autolysosome formation. Cell Structure and Function 27 29-37. (doi:10.1247/csf.27.29)

Nho RS \& Hergert P 2014 IPF fibroblasts are desensitized to type I collagen matrix-induced cell death by suppressing low autophagy via 
aberrant Akt/mTOR kinases. PLoS ONE 9 e94616. (doi:10.1371/journal. pone.0094616)

Obata K1, Morland SJ, Watson RH, Hitchcock A, Chenevix-Trench G, Thomas EJ \& Campbell IG 1998 Frequent PTEN/MMAC mutations in endometrioid but not serous or mucinous epithelial ovarian tumors. Cancer Research 58 2095-2097.

Porter AG \& Janicke RU 1999 Emerging roles of caspase-3 in apoptosis. Cell Death and Different 6 99-104. (doi:10.1038/sj.cdd.4400476)

Pritts EA \& Taylor RN 2003 An evidence-based evaluation of endometriosis associated infertility. Endocrinology Metabolism Clinics of North America 32 653-667. (doi:10.1016/S0889-8529(03)00045-8)

Rizner TL 2009 Estrogen metabolism and action in endometriosis. Molecular and Cellular Endocrinology 307 8-18. (doi:10.1016/j. mce.2009.03.022)

Ryan IP, Schriock ED \& Taylor RN 1994 Isolation, characterization, and comparison of human endometrial and endometriosis cells in vitro. Journal of Clinical Endocrinology and Metabolism 78 642-649. (doi:10.1210/jcem.78.3.8126136)

Saiki S, Sasazawa Y, Imamichi Y, Kawajiri S, Fujimaki T, Tanida I, Kobayashi H, Sato F, Sato S, Ishikawa K et al. 2011 Caffeine induces apoptosis by enhancement of autophagy via Pl3K/Akt/mTOR/p70S6K inhibition. Autophagy 7 176-178. (doi:10.4161/auto.7.2.14074)

Sarbassov DD, Ali SM \& Sabatini DM 2005 Growing roles for the mTOR pathway. Current Opinion in Cell Biology 17 596-603. (doi:10.1016/j. ceb.2005.09.009)

Sato N, Tsunoda H, Nishida M, Morishita $\mathrm{Y}$, Takimoto $\mathrm{Y}$, Kubo T \& Noguchi M 2000a Loss of heterozygosity on 10q23.3 and mutation of the tumor suppressor gene PTEN in benign endometrial cyst of the ovary: possible sequence progression from benign endometrial cyst to endometrioid carcinoma and clear cell carcinoma of the ovary. Cancer Research $607052-7056$.

Sato N, Nishida M \& Noguchi M 2000b An approach to early genetic alterations in precancerous cells. Human Cell 13 103-108.

Singh BN, Kumar D, Shankar S \& Srivastava RK 2012 Rottlerin induces autophagy which leads to apoptotic cell death through inhibition of PI3K/
Akt/mTOR pathway in human pancreatic cancer stem cells. Biochemical Pharmacology 84 1154-1163. (doi:10.1016/j.bcp.2012.08.007)

Steck PA, Pershouse MA, Jasser SA, Yung WK, Lin H, Ligon AH, Langford LA, Baumgard ML, Hattier T, Davis T et al. 1997 Identification of a candidate tumour suppressor gene, MMAC1, at chromosome $10 q 23.3$ that is mutated in multiple advanced cancers. Nature Genetics 15 356-362. (doi:10.1038/ng0497-356)

Sun H, Enomoto T, Fujita M, Wada H, Yoshino K, Ozaki K, Nakamura T \& Murata Y 2001 Mutational analysis of the PTEN gene in endometrial carcinoma and hyperplasia. American Journal of Clinical Pathology 115 32-38. (doi:10.1309/7JX6-B9U9-3POR-EQNY)

Vercellini P, Trespidi L, Colombo A, Vendola N, Marchini M \& Crosignani PG 1993 A gonadotropin-releasing hormone agonist versus a low-dose oral contraceptive for pelvic pain associated with endometriosis. Fertility and Sterility 60 75-79. (doi:10.1016/S0015-0282(16)56039-7)

Wu YC, Wu WK, Li Y, Yu L, Li ZJ, Wong CC, Li HT, Sung JJ \& Cho CH 2009 Inhibition of macroautophagy by bafilomycin A1 lowers proliferation and induces apoptosis in colon cancer cells. Biochemical and Biophysical Research Communications 382 451-456. (doi:10.1016/j.bbrc.2009.03.051)

Yorimitsu T \& Klionsky DJ 2005 Autophagy: molecular machinery for selfeating. Cell Death and Differentiation 12 (Supplement 2) 1542-1552. (doi:10.1038/sj.cdd.4401765)

Zhang G, Hou X, Li Y \& Zhao M 2014 MiR-205 inhibits cell apoptosis by targeting phosphatase and tensin homolog deleted on chromosome ten in endometrial cancer Ishikawa cells. BMC Cancer 14440 (doi:10.1186/1471-2407-14-440).

Received 14 June 2016

First decision 23 July 2016

Revised manuscript received 19 September 2016

Accepted 10 October 2016 